\title{
Neutron-gamma flux and dose calculations for feasibility study of DISCOMS instrumentation in case of severe accident in a GEN 3 reactor
}

\author{
Mariya Brovchenko ${ }^{1}$, Isabelle Duhamel ${ }^{1}$, Benjamin Dechenaux ${ }^{1}$ \\ ${ }^{1}$ Institut de Radioprotection et de Sûreté Nucléaire (IRSN), Fontenay-aux-Roses, 92260, France
}

\begin{abstract}
The present paper presents the study carried out in the frame of the DISCOMS project, which stands for "DIstributed Sensing for COrium Monitoring and Safety". This study concerns the calculation of the neutron and gamma radiations received by the considered instrumentation during the normal reactor operation as well as in case of a severe accident for the EPR reactor, outside the reactor pressure vessel and in the containment basemat. This paper summarizes the methods and hypotheses used for the particle transport simulation outside the vessel during normal reactor operation. The results of the simulations are then presented including the responses for distributed Optical Fiber Sensors (OFS), such as the gamma dose and the fast neutron fluence, and for Self Powered Neutron Detectors (SPNDs), namely the neutron and gamma spectra. Same responses are also evaluated for severe accident situations in order to design the SPNDs being sensitive to the both types of received neutron-gamma radiation. By contrast, fibers, involved as transducers in distributed OFS have to resist to the total radiation gamma dose and neutron fluence received during normal operation and the severe accident.
\end{abstract}

\section{Introduction}

At Fukushima Daiichi, the loss of electricity power supplies has quickly led most of instrumentation to be inoperative and the operator (TEPCO) with no way to monitor the status and the progress of the accident. To overcome these important drawbacks, the DISCOMS project [1], which stands for "DIstributed Sensing for COrium Monitoring and Safety", considers taking advantage of the potential of distributed sensing technologies based on both "Optical Fiber Sensors" (Raman, Brillouin, and Rayleigh Reflectometry) and long-length "Self Powered Neutron Detectors" (SPNDs).

The goal consists in inquiring about the status of the third barrier of confinement and to define possible mitigation strategies in case of severe accident, namely: i) reactor pressure vessel breakthrough and corium relocation outside the vessel, ii) concrete floor erosion and iii) corium cooling. Such monitoring should consist in "sensing cables" inserted in basemat below the reactor vessel and interrogated from a rear base, where operators can work safely. Thus, optical fibers selected for their resistance to ionizing radiations and long-length SPNDs, both judiciously deployed within the reactor basemat, and the structures around it, will provide a useful realtime or on-demand monitoring, during normal operation as well as in accidental and post-accidental situations.

For GEN 3 reactors (e.g. EPR reactor), distributed sensors (OFS and SPND) could be implemented below the reactor pressure vessel close to the surface of the reactor building basemat concrete and, if technically feasible, embedded into the concrete. The SPNDs need a sufficient level of radiation to generate a signal, and could be placed on the surface of the reactor pit.

This paper discusses the contribution to the development of remote monitoring technologies to improve Nuclear Power Plants (NPPs) safety. The work presented in this paper aims to provide the radiation level in terms of fast neutron fluence and the gamma dose for the optical fibers in order to ensure their resistance during the reactor life. Additionally, the SPNDs should be optimized in order to be sensitive to neutron and/or gamma flux during the normal operation of the reactor and during the severe accident (i.e. accident involving serious core damage) by calculating neutron and gamma flux spectra.

\section{Normal reactor operation}

To evaluate the neutron and gamma fluxes outside the vessel and in the basemat during normal reactor operation, the Monte Carlo code MCNP [2], which allows a detailed 3D geometrical description of the reactor core, the internals in the vessel and the reactor pit, was used with the associated ENDF/B-VII.1 nuclear data library.

The radiation source in this problem being the fissions occurring in the core of the reactor, the flux of

\footnotetext{
${ }^{\mathrm{a}}$ Corresponding author: mariya.brovchenko@irsn.fr
} 
neutral particles is strongly attenuated, especially in the basemat. Therefore, such neutron-gamma shielding calculations require the use of variance reduction (VR) methods.

\subsection{Variance reduction methods}

The authors used the variance reduction techniques implemented in the MCNP code, based on the Weight Window and source biasing.

In such problems, the positions of the responses (side of the vessel, vessel bottom, within the basemat) and the physical quantities (gamma dose, fast neutrons and thermal neutrons) are multiple. Optimizing a unique Weight Window set of maps (neutrons and gammas for different energy groups) to all of the responses is a challenge.

For this reason, Kenneth W. Burn (ENEA, Bologna) assisted the authors in the calculation of the VR parameters with a detailed mathematical approach called the DSA (Direct Statistical Approach) that optimizes the VR parameters governing the sampling [3]. The approach has been extended to include, among other things, weight control and a multiresponse capability [4].

\subsection{Source term}

The only source term considered in these calculations relies on the neutrons coming from fissions. As can be observed from the results, the gamma radiation from the irradiated fuel can be easily neglected. Nevertheless, the activation of structures outside the core is not taken into account in this study and could be not negligible.

The fission neutrons source distribution used in this study is a flat distribution within each assembly. Thus, the decrease of the neutron flux on the border of the assemblies on the edge of the core, as shown in figure 1 , is not modeled.

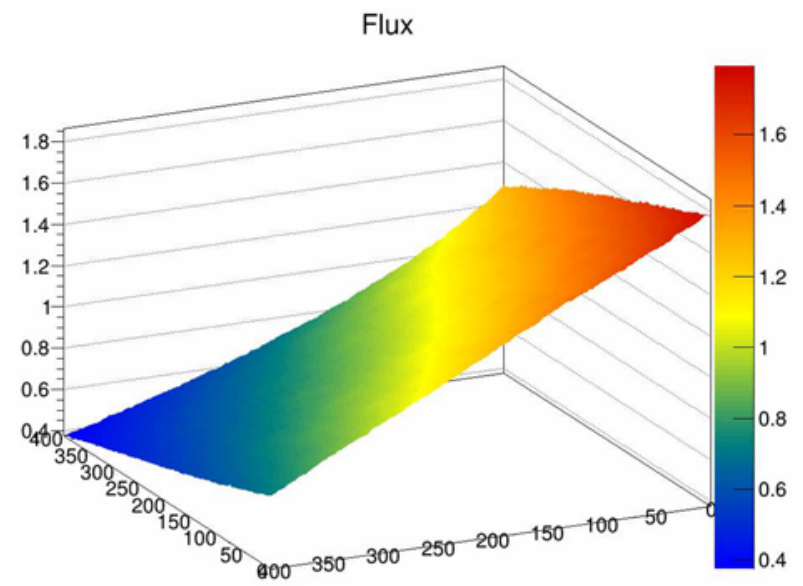

Figure 1. Flux distribution of an assembly on the edge of the core (arbitrary units)

Since only the first rows of assemblies on the edge of the core contribute to the flux estimation on or outside the vessel, the impact of this assumption was quantified. It leads to an overestimation of neutron and gamma fluxes outside the vessel of about $20 \%$ and $30 \%$ respectively.

\subsection{Reactor model}

Thanks to the MCNP code, detailed model of EPR reactor can be used for these calculations. The fuel compositions correspond to those of the end of an equilibrium cycle and are unique inside each assembly. The poisoned rods are treated separately.

No axial burn-up effect is taken into account. The geometry of the assemblies is detailed pin-by-pin (see figure 2).

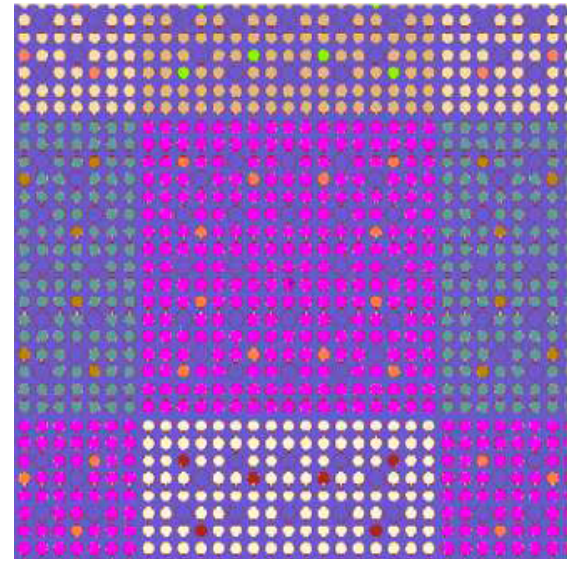

Figure 2. MCNP model of the pin-by-pin geometry of the assemblies

The geometry outside the core is modeled as precisely as possible. The positions of the responses of interest are presented in figures 3 and 4 . The detectors are placed outside the vessel at the level of the active core. The responses are calculated with an axial segmentation, as detailed in figure 3.

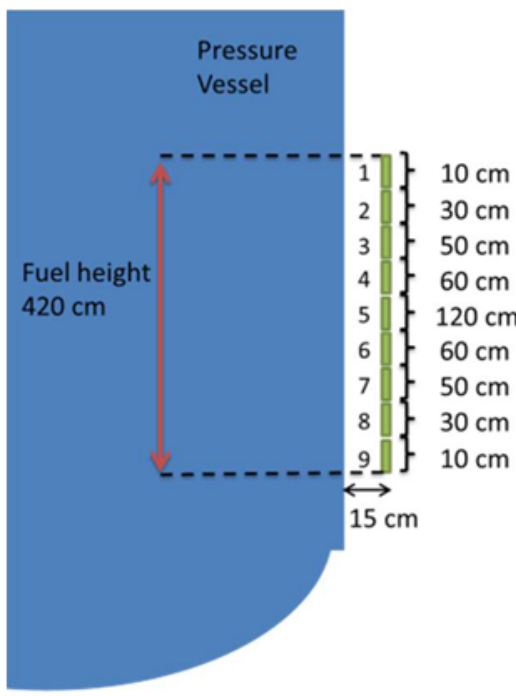

Figure 3. Positions of the responses on the side of the vessel with an axial segmentation 
The radiation is also studied under the vessel bottom on the basemat surface and at different depths inside the basemat. In the EPR reactor [5], it consists of a layer of sacrificial concrete (in green in figure 4) and a layer of refractory material (in blue in figure 4). As shown in figure 4 , the reactor pit is divided into 3 rings.

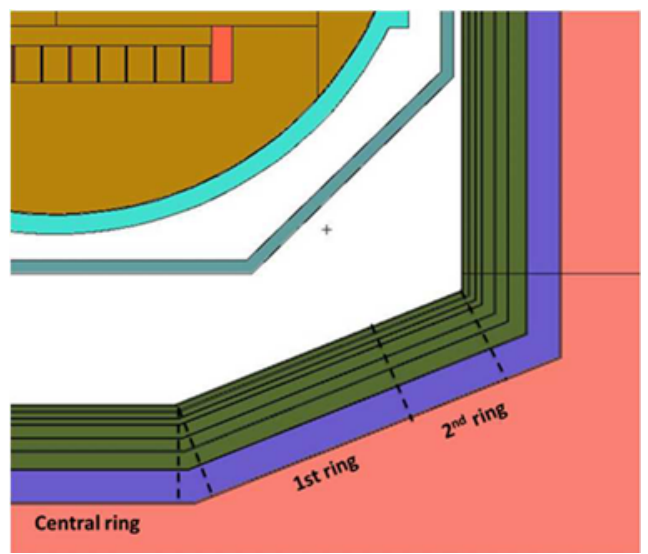

Figure 4. Positions of the responses in the reactor pit with a radial segmentation in rings

\section{Severe accident situation}

Several situations corresponding to different stages during a severe accident were considered in this study. The corium composition was calculated according to the hypotheses taken for each situation. Only the state with the spread corium in the reactor pit is presented here, in order to compare the radiation in the concrete to the one received during normal reactor operation.

\subsection{Corium}

The isotopic fuel composition is taken at the end of an equilibrium cycle, 3 hours after the reactor shut down, i.e. fissions stop. For sensitivity study, another corium composition was also considered supposing 49 hours after the reactor shut down. The fission products are supposed being not released from the corium. However, a sensitivity study was performed considering an important fission products release.

The corium model contains all the fuel mass, melted structural materials in the core and the vessel bottom. The composition is homogeneous and spread in the reactor pit, no corium-concrete interactions are considered.

\subsection{Source term and transport}

The source term considered here takes into account only the gamma rays issued from decay of instable isotopes found in the fuel composition. The spontaneous fissions activity was evaluated to be negligible $\left(10^{-9}\right.$ times lower than the total corium activity). The gamma rays source term is calculated using MCNP6 code and its new capabilities based on 25 energy groups emission data to generate the decay gamma rays. The decay heat evaluated by MCNP6 (tallying the energy deposition) was compared to the one given by the VESTA code [6] (using directly the decay energy from the JEFF 3.1 nuclear data library) resulting in a deviation of $13 \%$ on the decay heat. This deviation is acceptable for the purpose of its application here, since the calculation scheme is simplified: the source term and the transport can be performed in a single calculation.

The MCNP model, as presented in figure 5, is based on the same structures outside the vessel as used for the normal operation calculations. The positions of the responses in the reactor pit are the same as shown in figure 4. The corium is modeled as a cell with homogeneous composition and thus homogeneous source term.

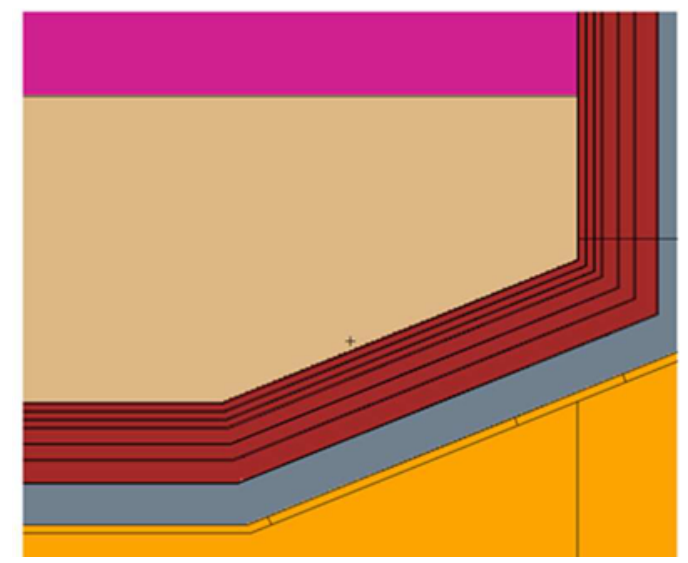

Figure 5. Corium (in beige) spread in the reactor pit on the sacrificial concrete (in red)

\section{Results}

\subsection{Neutrons}

The neutron responses were only calculated for the reactor full power operation state, since the spontaneous fission activity was negligible in the corium.

The responses were calculated using the VR parameters created with the DSA and converted to a set of classical Weight Windows. The transport calculation was performed using $3 \cdot 10^{8}$ source particles leading to a standard deviation of each response mostly lower than $1 \%$. Results are reported in the figures below.

The neutron flux spectrum calculated at the different positions on the side of the vessel and on the basemat surface is presented in figure 6 . The neutron flux varies by a factor 3 between the central axial segment and the bottom segment. The neutron energy spectrum is thereby unchanged.

The neutron flux on the surface of the basemat is more than one order of magnitude lower than on the side of the vessel, reaching 3 orders of magnitude for fast neutrons $(\mathrm{E}>1 \mathrm{MeV})$. The neutron flux on the outer rings of the basemat surface is slightly higher than in the central ring, which is mainly due to the shielding by the bottom of the vessel. Indeed, as verified by flagging the 
surfaces in the MCNP tallies, the contribution of particles that crosses the bottom of the vessel is very low.

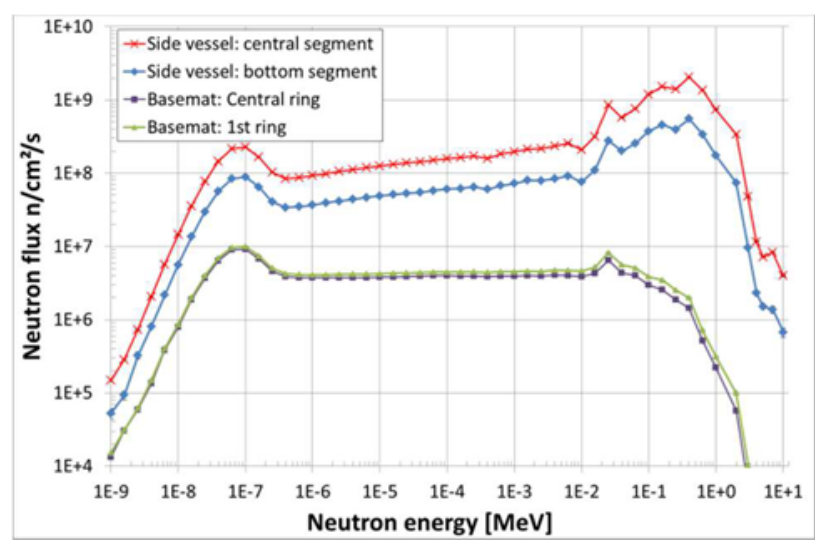

Figure 6. Neutron flux spectrum at different positions

Optical fiber sensors become opaque when subjected to neutron and gamma radiations. To protect them from the strong radiation in the nuclear reactor, the sensors can be embedded in the concrete or placed behind the refractory material layer.

To optimize the position, the neutron flux was evaluated at different depths in the reactor pit, considering 3 energy groups, as presented in the figure 7 . Thereby, strong hypothesis was made: the flux, calculated here for the core corresponding to an end of the equilibrium cycle, is considered constant during the whole reactor lifetime. This hypothesis is nevertheless in accordance to the purpose of these evaluations to provide an order of magnitude of the radiation level at different positions.

As shown in figure $7,50 \mathrm{~cm}$ of sacrificial concrete can provide an attenuation factor 100 for high $(\mathrm{E}>1 \mathrm{MeV})$ and low energy $(\mathrm{E}<0.1 \mathrm{MeV})$ neutron flux and a factor 10000 for intermediate energy neutron flux $(0.1 \mathrm{MeV}<\mathrm{E}<1 \mathrm{MeV}) .25 \mathrm{~cm}$ of refractory material provides an additional attenuation factor around 10 for all energy groups. It is to notice that the uncertainty becomes more important for values behind the refractory material, especially for the high energy neutrons.

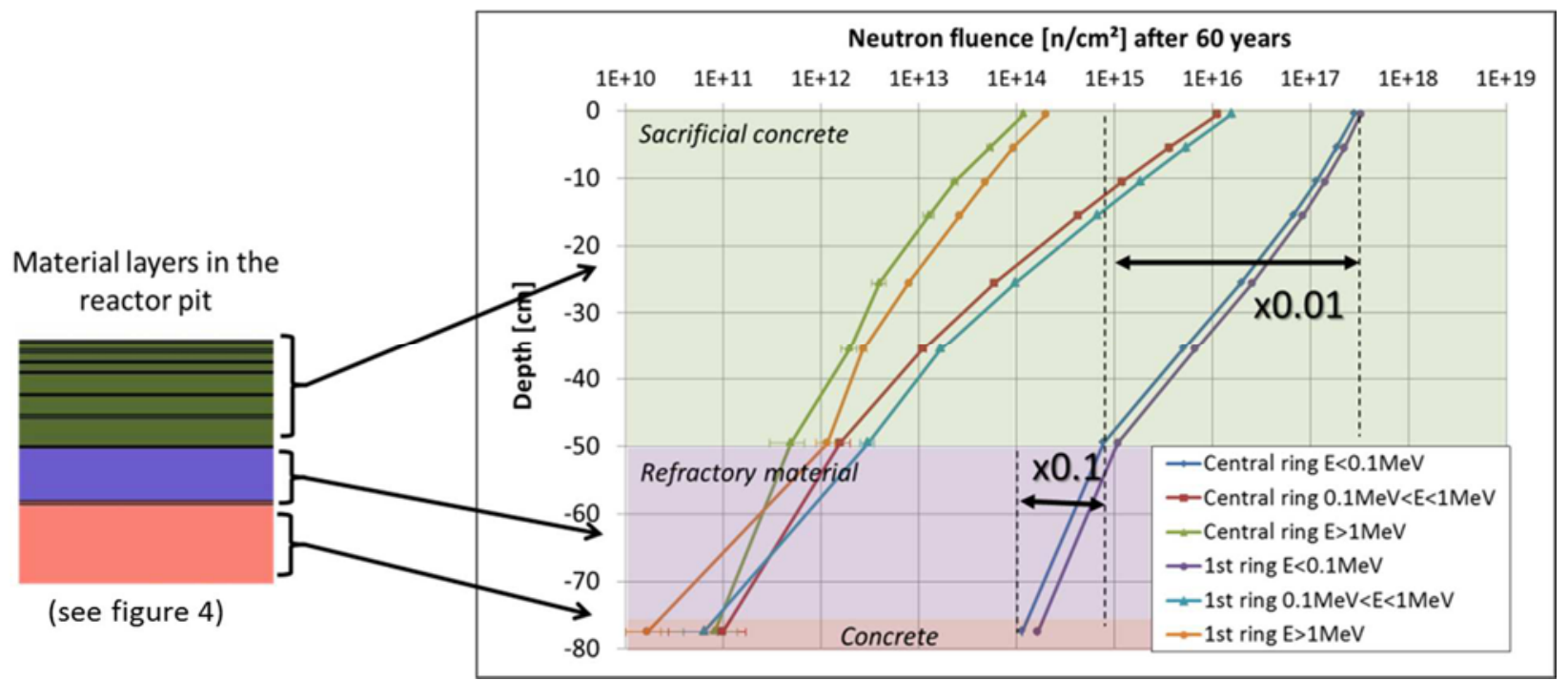

Figure 7. Neutron fluence attenuation considered for a constant flux during 60 years of reactor operation in the reactor pit divided in 3 energy groups in the different layers in the reactor pit

\subsection{Gamma rays}

For gamma radiation, the fluxes and doses were evaluated for the reactor operation at full power and the severe accident situations. The considered exposure time for the severe accident situation was 24 hours.

Figure 8 shows the gamma flux spectrum at different positions on the side of the vessel and on the surface of the basemat during normal reactor operation. Similarly to thermal neutrons, the gamma flux varies by a factor 3 between the central axial segment and the bottom segment and by more than one order of magnitude on the surface of the basemat. The gamma energy spectrum is almost unchanged even for the responses on the surface of the basemat.

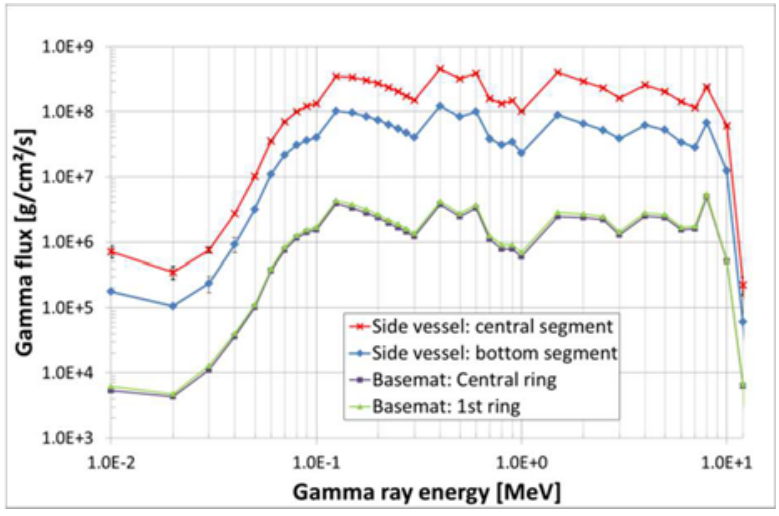

Figure 8. Gamma flux spectrum at different positions 
To quantify the potential protection of the optical fibers from gamma radiation in the concrete the attenuation of the kerma in silica was calculated. The results are presented in figure 9. The attenuation is not exponential since the gamma rays are also produced by the neutrons directly in the basemat. Due to this, the sacrificial concrete provides only an attenuation of a factor 20. However, $25 \mathrm{~cm}$ of refractory material attenuates the kerma by an additional factor 100 .

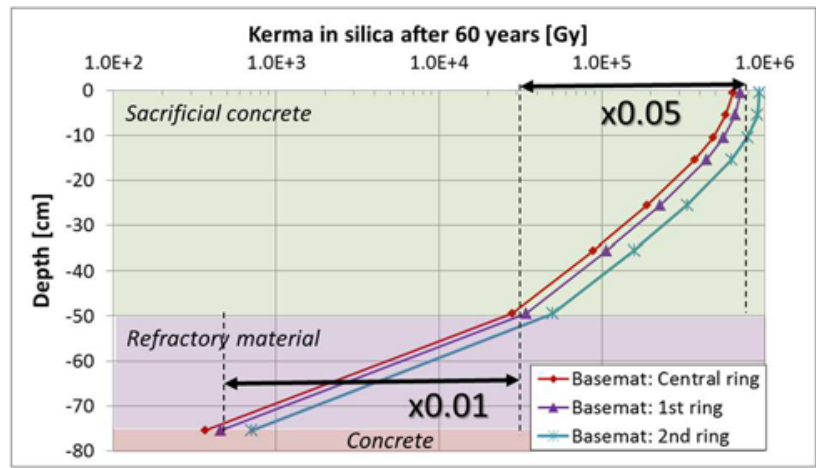

Figure 9. Gamma kerma in silica attenuation in the basemat considered for a constant flux during 60 years of reactor operation

For the severe accident situation, as described above, the same gamma responses in the basemat were calculated. The gamma ray spectrum was calculated on the concrete surface, directly in contact with the corium. The spectrum is compared to the one obtained during reactor operation at the same position and is presented in figure 10. The flux is 10000 times higher in contact with the corium compared to the flux received during operation. The relative proportion of the high energy gamma rays (1-10 MeV) coming from the corium is low especially for gamma rays with $\mathrm{E}>3 \mathrm{MeV}$. This part of the spectrum is very different from the one observed for gamma ray flux received during reactor operation due to the fact that the gamma rays issued from the neutron reactions have higher energies than the ones issued from the decays.

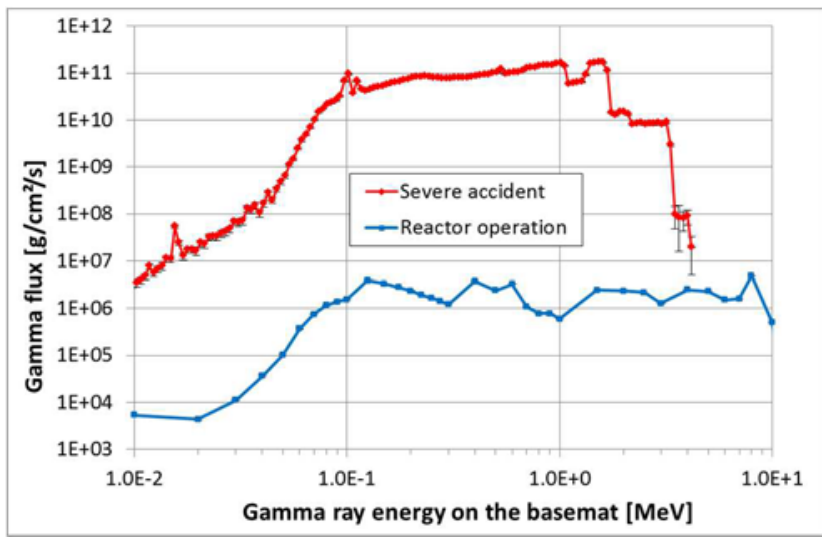

Figure 10. Gamma flux spectrum received in severe accident and during reactor operation on the surface of the basemat
The attenuation of the kerma in the basemat is shown in figure 11. For a gamma source, the concrete of $50 \mathrm{~cm}$ thickness provides a good protection with an attenuation of a factor 1000. An additional factor 1000 can also be provided by the refractory material $(25 \mathrm{~cm}$ thickness).

With the hypothesis that the Optical Fiber Sensors have to resist at least for 24 hours after the accident onset, the choice of their position in the sacrificial concrete is driven by the gamma radiation received during normal reactor operation, as shown in figure 11 . Indeed, in the first $5 \mathrm{~cm}$ of the concrete the gamma kerma from corium is more important than the one due to normal reactor operation, but at this distance from corium the sensor resistance to high temperatures is the dominant issue.

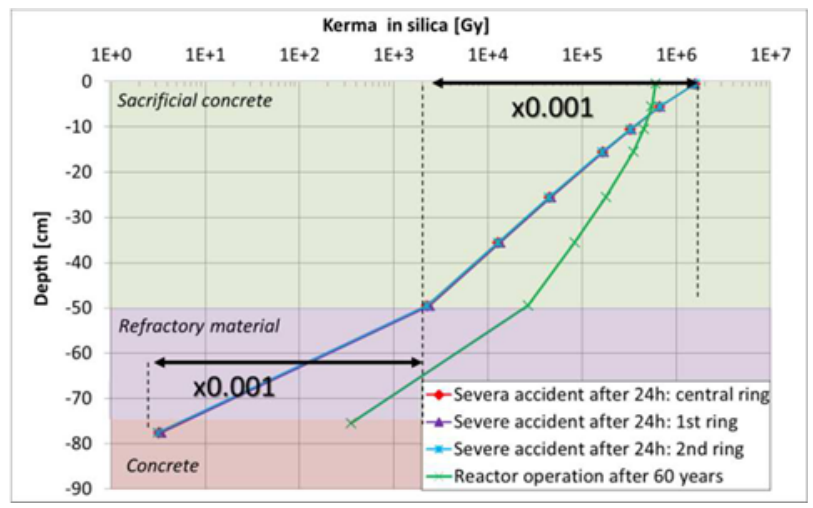

Figure 11. Gamma kerma in silica after 24 hours with corium on the basemat and after 60 years of normal operation

Due to the nature of a severe accident situation, assumptions must be made. In order to test the impact of these assumptions, some sensitivity calculations were performed.

The assumption for the corium isotopic composition of 3 hours "cooling time" after the reactor shut down was compared with 49 hours. The impact is a decrease of the kerma by around $30 \%$, and a small change in the gamma ray spectrum.

The impact of the presence of volatile and halfvolatile fission products in the corium was calculated. Two corium compositions were compared: one keeping all the fission products in the corium and another one considering an important release of volatile and semivolatile fission products. The comparison showed a decrease of kerma by $30-35 \%$, and a small change in the gamma ray spectrum.

Another important assumption is to consider that the corium composition is homogenous. Nevertheless, the impact of this hypothesis was not studied here.

\section{Conclusion}

In the framework of the DISCOMS project, the feasibility of the two types of instrumentation, Optical Fiber Sensors and SPNDs, is studied to contribute to the NPP safety improvement. The neutron and gamma fluxes 
and spectra were calculated at different positions outside the reactor vessel and on the basemat. The radiation was characterized for a state during normal reactor operation and severe accident situation. These results contribute to the design definition and the possible location choice for SPNDs with the aim that the SPNDs can provide a valuable signal for the both type of states.

To protect the optical fibers from radiation damages, fast neutron fluence and gamma kerma were calculated. For this, both types of situation were considered, the radiation received during reactor normal operation and the one received during the severe accident. As a result, the authors can conclude that the radiation damage to Optical Fiber Sensors will be mainly driven by the radiation received during reactor normal operation, regarding neutrons and gamma rays. In parallel to the radiation level calculations, the sensors designer should consider the temperature distribution and its evolution during the severe accident. The radiation and temperature "maps" should allow the DISCOMS project partners to select the most appropriate sensors' position and the instrumentation design.

\section{Acknowledgment}

This project is co-funded by the French "Programme d'Investissements d'Avenir (PIA)" referenced by $\mathrm{n}^{\circ}$ ANR-11-RSNR-0007 and managed by the ANR (National Research Agency).

The authors would like to thank all the partners of the DISCOMS project, especially AREVA for the data supplied concerning the EPR modeling and instrumentation specification but also colleagues from the CEA involved in both Optical Fiber Sensors and SPNDs developments, for all the technical exchanges and fruitful discussions on these instrumentations.

Authors' particular thanks go to Kenneth W. Burn (ENEA), for his huge help in performing calculations of radiation during reactor operation, for his advice and for sharing his experience.

\section{References}

1. P. Ferdinand et al., "DISCOMS: DIstributed Sensing for COrium Monitoring and Safety", CANSMART 2015: International Conference on Smart Materials and Structures, CANSMART, 15-17 July, Vancouver, $B C$, Canada (2015)

2. T. Goorley et al., "Initial MCNP6 Release Overview MCNP6 version 1.0", LA-UR-13-22934, (2013)

3. A. Dubi et al., "General Statistical Model for Geometrical Splitting in Monte Carlo - parts I and II", Transp. Theory Stat. Phys. 14(2), 167-193 and 195-221 (1985)

4. K.W. Burn, "Optimizing Monte Carlo to multiple responses: the Direct Statistical Approach, 10 years on", Nuclear Technology 175, 138-145 (2011)

5. M. Fischer, "The severe accident mitigation concept and the design measures for core melt retention of the
European Pressurized Reactor (EPR)", Nuclear Engineering and Design 230 169-180 (2004)

6. W. Haeck, "VESTA User's Manual - Version 2.1.0", IRSN DSU/SEC/T/2011-81, Institut de Radioprotection et de Sûreté Nucléaire, France (2012) 